\title{
SOME PROPERTIES OF GEODESIC STRONGLY E-B-VEX FUNCTIONS
}

\author{
WEDAD SALEH* \\ Department of Mathematics, Taibah University, Al- Medina, Saudi Arabia \\ *Corresponding author: wed_10_777@hotmail.com
}

\begin{abstract}
Geodesic E-b-vex sets and geodesic E-b-vex functions on a Riemannian manifold are extended to the so called geodesic strongly E-b-vex sets and geodesic strongly E-b-vex functions. Some basic properties of geodesic strongly E-b-vex sets are also studied.
\end{abstract}

\section{INTRODUCTION}

Convexity and its generalizations play an important role in optimization theory, convex anlysis and Minkowski space $[3,4,6,9,10]$.

Youness [17] defined E-convex sets and E-convex functions by relaxing the definitions of convex sets and convex functions, which have some important applications in various branches of mathematical sciences $[1,12,13]$. Also, Youness [18] extended the definitions of E-convex sets and E-convex functions to strongly E-convex sets and strongly E-convex functions. The B-vex functions which shares many properties with convex functions was introduced by Bector and Singh [2]. Some reserchers studied some new generalizations of convex functions by relaxing definitions of E-convex functions and B-vex functions such as E-B-vex functions [15] and strongly E-B-vex functions [19]. Also, generalization of convexity on Riemannian manifolds were presented in ( [5], [8], [14], [16]).

Received 2019-03-08; accepted 2019-04-05; published 2019-05-01.

2010 Mathematics Subject Classification. 52A20, 52A41, 53C20, 53C22.

Key words and phrases. geodesic E-convex sets; geodesic E-convex functions; Riemannian manifolds.

(C)2019 Authors retain the copyrights of their papers, and all open access articles are distributed under the terms of the Creative Commons Attribution License. 
In this paper, a new class of sets on Riemannian manifolds, called geodesic strongly E-b-vex sets, and a new class of functions defined on them, called geodesic strongly E-convex functions, have been proposed. Also, some of their properties have been discussed. This paper divides into three sections. In section 2 , some of definities and properties which will be used throughout this work are presented that can be found in many books on differential geometry such as [16]. In section 3, a geodedic strongly E-b-vex set and geodesic strongly E-b-vex function are studied with some of their properties.

\section{Preliminaries}

Now, let $\aleph$ is a $C^{\infty}$ n-dimensional Riemannian manifold, also $\mu_{1}, \mu_{2} \in \aleph$ and $\delta:[0,1] \longrightarrow \aleph$ be a geodesic joining the points $\mu_{1}$ and $\mu_{2}$, which means that $\delta_{\mu_{1}, \mu_{2}}(0)=\mu_{2}$ and $\delta_{\mu_{1}, \mu_{2}}(1)=\mu_{1}$.

Strongly E-convex sets (SEC) and strongly E-convex (SEC) functions were introduced in [18] such as:

Definition 2.1. (1) A subset $\Omega \subseteq \mathbb{R}^{n}$ is strongly E-convex (SEC) set if there is a map $\varepsilon: \mathbb{R}^{n} \longrightarrow \mathbb{R}^{n}$ such that

$$
\delta\left(\alpha \mu_{1}+\varepsilon\left(\mu_{1}\right)\right)+(1-\delta)\left(\alpha \mu_{2}+\varepsilon\left(\mu_{2}\right)\right) \in B
$$

for each $\mu_{1}, \mu_{2} \in \Omega, \alpha \in[0,1]$ and $\delta \in[0,1]$.

(2) A function $g: \Omega \subseteq \mathbb{R}^{n} \longrightarrow \mathbb{R}$ is strongly E-convex (SEC) function on $\Omega$ if there is a map $\varepsilon: \mathbb{R}^{n} \longrightarrow \mathbb{R}^{n}$ such that $\Omega$ is a SEC set and

$$
g\left(\delta\left(\alpha \mu_{1}+\varepsilon\left(\mu_{1}\right)\right)+(1-\delta)\left(\alpha \mu_{2}+\varepsilon\left(\mu_{2}\right)\right)\right) \leq \delta g\left(\varepsilon\left(\mu_{1}\right)\right)+(1-\delta) g\left(\varepsilon\left(\mu_{2}\right)\right),
$$

$\forall \mu_{1}, \mu_{2} \in \Omega, \alpha \in[0,1]$ and $\delta \in[0,1]$

Definition 2.2. [5]

(1) Considering $\varepsilon: \aleph \longrightarrow \aleph$ is a map. A subset $\Omega \subset \aleph$ is geodesic E-convex iff there exists a unique geodesic $\eta_{\varepsilon\left(\mu_{1}\right), \varepsilon\left(\mu_{2}\right)}(\delta)$ of length $d\left(\mu_{1}, \mu_{2}\right)$, which belongs to $\Omega, \forall \mu_{1}, \mu_{2} \in \Omega$ and $\delta \in[0,1]$.

(2) A functin $g: \Omega \subseteq \aleph \longrightarrow \mathbb{R}$ where $\Omega$ is a GEC set in $\aleph$ is geodesic E-convex if

$$
g\left(\eta_{\varepsilon\left(\mu_{1}\right), \varepsilon\left(\mu_{2}\right)}(\delta)\right) \leq \delta g\left(\varepsilon\left(\mu_{1}\right)\right)+(1-\delta) g\left(\varepsilon\left(\mu_{2}\right)\right)
$$

$\forall \mu_{1}, \mu_{2} \in \Omega$ and $\delta \in[0,1]$

\section{Definition 2.3. $[7]$}

(1) Considering $\varepsilon: \aleph \longrightarrow \aleph$ is a map. A subset $\Omega \subset \aleph$ is geodesic strongly E-convex(GSEC) iff there exists a unique geodesic

$\eta_{\alpha \mu_{1}+\varepsilon\left(\mu_{1}\right), \alpha \mu_{2}+\varepsilon\left(\mu_{2}\right)}(\delta)$ of length $d\left(\mu_{1}, \mu_{2}\right)$, which belongs to $\Omega, \forall \mu_{1}, \mu_{2} \in \Omega, \alpha \in[0,1]$ and $\delta \in[0,1]$ and . 
(2) A functin $g: \Omega \subseteq \aleph \longrightarrow \mathbb{R}$, where $\Omega$ is a GSEC set in $\aleph$, is geodesic strongly E-convex (GSEC) funtion if

$$
g\left(\eta_{\alpha \mu_{1}+\varepsilon\left(\mu_{1}\right), \alpha \mu_{2}+\varepsilon\left(\mu_{2}\right)}(\delta)\right) \leq \delta g\left(\varepsilon\left(\mu_{1}\right)\right)+(1-\delta) g\left(\varepsilon\left(\mu_{2}\right)\right),
$$

$\forall \mu_{1}, \mu_{2} \in \Omega$ and $\delta \in[0,1]$.

\section{Geodesic Strongly E-B-vex Sets and Geodesic Strongly E-B-vex Functions}

In this part of work, a geodesic strongly E-b-vex (GSE-b-vex) set and a geodesic strongly E-b-convex (GSE-b-vex) function in a Riemannian manifold $\aleph$ are given and some of their properties are discussed.

Definition 3.1. A subset $\Omega$ of $\aleph$ is called a geodesic strongly E-b-vex (GSE-b-vex) iff there exists a unique geodesic $\eta_{\alpha \mu_{1}+\varepsilon\left(\mu_{1}\right), \alpha \mu_{2}+\varepsilon\left(\mu_{2}\right)}(\delta b)$ of length $d\left(\mu_{1}, \mu_{2}\right)$, which belongs to $\Omega, \forall \mu_{1}, \mu_{2} \in \Omega, \alpha \in[0,1]$ and $\delta \in[0,1]$.

Remark 3.1. (1) Every GSE-b-vex set is a GSEC set when $b\left(\mu_{1}, \mu_{2}, \delta\right)=1$.

(2) Every GSE-b-vex set is a GE-b-vex set when $\alpha=0$.

(3) When

$$
\eta_{\alpha \mu_{1}+\varepsilon\left(\mu_{1}\right), \alpha \mu_{2}+\varepsilon\left(\mu_{2}\right)}(\delta b)=\delta b\left(\alpha \mu_{1}+\varepsilon\left(\mu_{1}\right)\right)+(1-\delta b)\left(\alpha \mu_{2}+\varepsilon\left(\mu_{2}\right)\right),
$$

then we have strongly E-B-vex set.

Now, some propertie of GSE-b-vex sets are propoed.

Proposition 3.1. Every convex set $\Omega \subset \aleph$ is a GSE-b-vex set.

The proof of the above proposition is direct that by taking $\varepsilon: \aleph \longrightarrow \aleph$ as the identity map, $b\left(\mu_{1}, \mu_{2}, \delta\right)=1$ and $\alpha=0$.

Proposition 3.2. Let $\Omega \subset \aleph$ be a GSE-b-vex set, then $\varepsilon(\Omega) \subseteq \Omega$.

Proof. Since $\Omega$ is a GSE-b-vex set, then

$$
\eta_{\alpha \mu_{1}+\varepsilon\left(\mu_{1}\right), \alpha \mu_{2}+\varepsilon\left(\mu_{2}\right)}(\delta b) \in \Omega,
$$

$\mu_{1}, \mu_{2} \in \Omega, \alpha \in[0,1]$ and $\delta \in[0,1]$. Let $\delta b=1$ and $\alpha=0$, then $\eta_{\varepsilon\left(\mu_{1}\right), \varepsilon\left(\mu_{2}\right)}=\varepsilon\left(\mu_{2}\right) \in \Omega$, then $\varepsilon(\Omega) \subseteq \Omega$.

Theorem 3.1. Suppose that a set $\left\{\Omega_{j}\right\}_{j=1,2, \cdots, n}$ is an arbitrary collection of GSE-v-vex subsets of $\aleph$, then $\cap_{j=1,2, \cdots, n} \Omega_{i}$ is a GSE-b-vex set.

Proof. Considering $\left\{\Omega_{j}\right\}_{j=1,2, \cdots, n}$ is a collection of GSE-b-vex subsets of $\Omega$. If $\cap_{j=1,2, \cdots, n} \Omega_{j}$ is an empty set, then the result is obvious. Assume that $\mu_{1}, \mu_{2} \in \cap_{j=1,2, \cdots, n} \Omega_{j}$, then $\mu_{1}, \mu_{2} \in \Omega_{j}$. Hence, $\eta_{\alpha \mu_{1}+\varepsilon\left(\mu_{1}\right), \alpha \mu_{2}+\varepsilon\left(\mu_{2}\right)}(\delta b) \in$ $\Omega_{j}, \forall \alpha \in[0,1]$ and $\delta \in[0,1]$. Hence, $\eta_{\alpha \mu_{1}+\varepsilon\left(\mu_{1}\right), \alpha \mu_{2}+\varepsilon\left(\mu_{2}\right)}(\delta b) \in \cap_{j=1,2, \cdots, n} \Omega_{j}, \forall \alpha \in[0,1]$ and $\delta \in[0,1]$. 
Remark 3.2. However, the above theorem is not true for the union of GSE-b-vex sets.

Now, we introduce the definition of a geodesic E-b-vex (GSE-b-vex) function on $\aleph$.

Definition 3.2. Assume that $\Omega \subset \aleph$ is a GSE-b-vex set. A function $g: \Omega \longrightarrow \mathbb{R}$ is called a geodesic strongly E-b-vex (GSE-b-vex) if

$$
g\left(\eta_{\alpha \mu_{1}+\varepsilon\left(\mu_{1}\right), \alpha \mu_{2}+\varepsilon\left(\mu_{2}\right)}(\delta b)\right) \leq \gamma g\left(\varepsilon\left(\mu_{1}\right)\right)+(1-\gamma) g\left(\varepsilon\left(\mu_{2}\right)\right),
$$

$\forall \mu_{1}, \mu_{2} \in \Omega, \alpha \in[0,1]$ and $\delta \in[0,1]$.

If the inequality (3.1) is strict, then $g$ is called a strictly GSE-b-vex function.

Example 3.1. Assume that $g: \mathbb{R} \longrightarrow \mathbb{R}$ such that $g(\mu)=-|\mu|$. Aslo, assume that $\varepsilon: \mathbb{R} \longrightarrow \mathbb{R}$ is defined as $\varepsilon(\mu)=\alpha \mu$ where $0<\alpha \leq 1, \forall \mu \in \mathbb{R}$ and the geodesic $\eta$ is given as

$$
\begin{aligned}
& \eta_{\alpha \mu_{1}+\varepsilon\left(\mu_{1}\right), \alpha \mu_{2}+\varepsilon\left(\mu_{2}\right)}(\delta b) \begin{cases}\frac{1}{2 \alpha}\left[\alpha \mu_{2}+\varepsilon\left(\mu_{2}\right)+\delta b\left(\alpha \mu_{1}+\varepsilon\left(\mu_{1}\right)-\alpha \mu_{2}-\varepsilon\left(\mu_{2}\right)\right)\right] & ; \mu_{1} \mu_{2} \geq 0, \\
\frac{1}{2 \alpha}\left[\alpha \mu_{2}+\varepsilon\left(\mu_{2}\right)+\delta b\left(\alpha \mu_{2}+\varepsilon\left(\mu_{2}\right)-\alpha \mu_{1}-\varepsilon\left(\mu_{1}\right)\right)\right] & ; \mu_{1} \mu_{2}<0\end{cases} \\
&= \begin{cases}\mu_{2}+\delta b\left(\mu_{1}-\mu_{2}\right) & ; \mu_{1} \mu_{2} \geq 0, \\
\mu_{2}+\delta b\left(\mu_{2}-\mu_{1}\right) & ; \mu_{1} \mu_{2}<0,\end{cases}
\end{aligned}
$$

then $g$ is GSE-b-vex function.

Proposition 3.3. Let $g: \Omega \longrightarrow \mathbb{R}$ be a GSE-b-vex function on a GSE-b-vex set $\Omega \times \aleph$, then $g(\alpha \mu+\varepsilon(\mu)) \leq$ $g(\varepsilon(\mu)), \mu \in \Omega$ and $\alpha \in[0,1]$.

Proof. Since $g$ is GSE-b-vex function on GSE-b-vex set $\Omega$, then

$$
g\left(\eta_{\alpha \mu_{1}+\varepsilon\left(\mu_{1}\right), \alpha \mu_{2}+\varepsilon\left(\mu_{2}\right)}(\delta b)\right) \leq \delta b g\left(\varepsilon\left(\mu_{1}\right)\right)+(1-\delta b) g\left(\varepsilon\left(\mu_{2}\right)\right),
$$

then for $\delta b=1$, we have

$$
g\left(\alpha \mu_{1}+\varepsilon\left(\mu_{1}\right)\right) \leq g\left(\varepsilon\left(\mu_{1}\right)\right) .
$$

Theorem 3.2. If $g_{1}: \Omega \longrightarrow \mathbb{R}$ is a GSE-b-vex function on a GSE-b-vex set $\Omega \subset \aleph$ and $g_{2}: U \longrightarrow \mathbb{R}$ is a non-decreasing convex function such that rang $\left(g_{1}\right) \subset U$, then the composite function $g_{2} \circ g_{1}$ is GSE-b-vex function on $\Omega$. 
Proof. By using the hypothesis, we can write all $x_{1}, x_{2} \in B, \alpha \in[0,1]$ and $\gamma \in[0,1]$,

$$
g_{1}\left(\eta_{\alpha \mu_{1}+\varepsilon\left(\mu_{1}\right), \alpha \mu_{2}+\varepsilon\left(\mu_{2}\right)}(\delta b)\right) \leq \delta b g_{1}\left(\varepsilon\left(\mu_{1}\right)\right)+(1-\delta b) g_{1}\left(\varepsilon\left(\mu_{2}\right)\right)
$$

$\forall \mu_{1}, \mu_{2} \in \Omega, \alpha \in[0,1]$ and $\delta \in[0,1]$ and since $g_{2}$ is a non-decreasing convex function, then we get

$$
\begin{aligned}
g_{2} o g_{1}\left(\eta_{\alpha \mu_{1}+\varepsilon\left(\mu_{1}\right), \alpha \mu_{2}+\varepsilon\left(\mu_{2}\right)}(\delta b)\right) & =g_{2}\left(g_{2}\left(\eta_{\alpha \mu_{1}+\varepsilon\left(\mu_{1}\right), \alpha \mu_{2}+\varepsilon\left(\mu_{2}\right)}(\delta b)\right)\right) \\
& \leq g_{2}\left(\delta b g_{1}\left(\varepsilon\left(\mu_{1}\right)\right)+(1-\delta b) g_{1}\left(\varepsilon\left(\mu_{2}\right)\right)\right) \\
& \leq \delta b g_{2}\left(g_{1}\left(\varepsilon\left(\mu_{1}\right)\right)\right)+(1-\delta b) g_{2}\left(g_{1}\left(\varepsilon\left(\mu_{2}\right)\right)\right) \\
& =\delta b\left(g_{2} o g_{1}\right)\left(\varepsilon\left(\mu_{1}\right)\right)+(1-\delta b)\left(g_{2} o g_{1}\right)\left(\varepsilon\left(\mu_{2}\right)\right)
\end{aligned}
$$

hence, $g_{2} o g_{1}$ is GSE-b-vex on $\Omega$. Moreover, $g_{2} \circ g_{1}$ is a strictly GSE-b-vex function if $g_{2}$ is a strictly nondecreasing convex function.

Theorem 3.3. Considering $g_{i}: \Omega \longrightarrow \mathbb{R}, i=1,2, \ldots, n$ are GSE-b-vex functions. Then, the function

$$
g=\sum_{i=1}^{n} \xi_{i} g_{i}
$$

is also GSE-b-vex geodesic on $\Omega, \forall \xi_{i} \in \mathbb{R}, \xi_{i} \geq 0$.

Proof. Since $g_{i}, i=1,2, \ldots, n$ are GSE-b-vex functions, then

$$
g_{i}\left(\eta_{\alpha \mu_{1}+\varepsilon\left(\mu_{1}\right), \alpha \mu_{2}+\varepsilon\left(\mu_{2}\right)}(\delta b)\right) \leq \delta b g_{i}\left(\varepsilon\left(\mu_{1}\right)\right)+(1-\delta b) g_{i}\left(\varepsilon\left(\mu_{2}\right)\right),
$$

$\forall \mu_{1}, \mu_{2} \in \Omega, \alpha \in[0,1]$ and $\delta \in[0,1]$, Hence,

$$
\xi_{i} g_{i}\left(\eta_{\alpha \mu_{1}+\varepsilon\left(\mu_{1}\right), \alpha \mu_{2}+\varepsilon\left(\mu_{2}\right)}(\delta b)\right) \leq \delta b \xi_{i} g_{i}\left(\varepsilon\left(\mu_{1}\right)\right)+(1-\delta b) \xi_{i} g_{i}\left(\varepsilon\left(\mu_{2}\right)\right) .
$$

This implies to,

$$
\begin{aligned}
g\left(\eta_{\alpha \mu_{1}+\varepsilon\left(x_{1}\right), \alpha \mu_{2}+\varepsilon\left(\mu_{2}\right)}(\delta b)\right) & =\sum_{i=1}^{n} \xi_{i} g_{i}\left(\eta_{\alpha \mu_{1}+\varepsilon\left(x_{1}\right), \alpha \mu_{2}+\varepsilon\left(\mu_{2}\right)}(\delta b)\right) \\
& \leq \delta b \sum_{i=1}^{n} \xi_{i} g_{i}\left(\varepsilon\left(\mu_{1}\right)\right)+(1-\delta b) \sum_{i=1}^{n} \xi_{i} g_{i}\left(\varepsilon\left(\mu_{2}\right)\right) \\
& =\delta b g\left(\varepsilon\left(\mu_{1}\right)\right)+(1-\delta b) g\left(\varepsilon\left(\mu_{2}\right)\right) .
\end{aligned}
$$

Then $g$ is GSE-b-vex function.

Next, we show that a funcion is GSE-b-vex iff its epigraph is a GSE-b-vex set.

Definition 3.3. Assume that $\Omega \subset \aleph \times \mathbb{R}, E: \aleph \longrightarrow \aleph, b: \Omega \times \Omega \times[0,1] \longrightarrow \mathbb{R}_{+}$and $F: \mathbb{R} \longrightarrow \mathbb{R}$. A set $\Omega$ is called a geodesic strongly $E \times F$-convex $(G S E \times F$-b-vex $)$ if

$$
\left(\eta_{\alpha \mu_{1}+\varepsilon\left(\mu_{1}\right), \alpha \mu_{2}+\varepsilon\left(\mu_{2}\right)}(\delta b), \delta b F\left(\xi_{1}\right)+(1-\delta b) F\left(\xi_{2}\right)\right) \in \Omega
$$

$\forall\left(\mu_{1}, \xi_{1}\right),\left(\mu_{2}, \xi_{2}\right) \in \Omega, \alpha \in[0,1]$ and $\gamma \in[0,1]$. 
Remark 3.3. From Definition 3.3, we have found $\Omega \subseteq \aleph$ is a GSE-b-vex set iff $\Omega \times \mathbb{R}$ is a GSE $\times F$-b-vex set.

Now, the epigraph of a function $g: \Omega \subset \aleph \longrightarrow \mathbb{R}$ is given as

$$
E(g)=\{(\mu, a): \mu \in \Omega, a \in \mathbb{R}, g(\mu) \leq a\} .
$$

Theorem 3.4. Suppose that $\Omega \subseteq \aleph$ is a GSE-b-vex set, $g: \Omega \longrightarrow \mathbb{R}$ is a function and $F: \mathbb{R} \longrightarrow \mathbb{R}$ is a map such that $F(g(\mu)+a)=g(\varepsilon(\mu))+a, \forall a \in \mathbb{R}, a \geqslant 0$. Then, $g$ is a GSE-b-vex on $\Omega$ iff $E(g)$ is a GSE $\times F$-b-vex on $\Omega \times \mathbb{R}$.

Proof. Let $\left(\mu_{1}, a_{1}\right),\left(\mu_{2}, a_{2}\right) \in E(g)$. Since $\Omega$ is GSE-b-vex, then

$$
\eta_{\alpha \mu_{1}+\varepsilon\left(\mu_{1}\right), \alpha \mu_{2}+\varepsilon\left(\mu_{2}\right)}(\delta b) \in \Omega
$$

$\forall \alpha \in[0,1]$ and $\delta \in[0,1]$. When $\alpha=0$ and $\delta b=1$, we have $\varepsilon\left(\mu_{1}\right) \in \Omega$ also, when $\alpha=0$ and $\delta b=0$ we get $\varepsilon\left(\mu_{2}\right) \in \Omega$. Assume that $F\left(a_{1}\right)$ and $F\left(a_{2}\right)$ where $g\left(\varepsilon\left(\mu_{1}\right)\right) \leq F\left(a_{1}\right)$ and $g\left(\varepsilon\left(\mu_{2}\right)\right) \leq F\left(a_{2}\right)$. Then

$$
\left(\varepsilon\left(\mu_{1}\right), F\left(a_{1}\right)\right),\left(\varepsilon\left(\mu_{2}\right), F\left(a_{2}\right)\right) \in E(g) .
$$

Considering $g$ is a GSE-b-vex on $\Omega$, then

$$
\begin{aligned}
g\left(\eta_{\alpha \mu_{1}+\varepsilon\left(\mu_{1}\right), \alpha \mu_{2}+\varepsilon\left(\mu_{2}\right)}(\delta b)\right) & \leq \delta b g\left(\varepsilon\left(\mu_{1}\right)\right)+(1-\delta b) g\left(\varepsilon\left(\mu_{2}\right)\right) \\
& \leq \delta b F\left(a_{1}\right)+(1-\delta b) F\left(a_{2}\right) .
\end{aligned}
$$

This is leading to, $\left(\eta_{\alpha \mu_{1}+\varepsilon\left(\mu_{1}\right), \alpha \mu_{2}+\varepsilon\left(\mu_{2}\right)}(\delta b), \delta b F\left(a_{1}\right)+(1-\delta b) F\left(a_{2}\right)\right) \in E(g)$, which means that $E(g)$ is GSE $\times \dot{E}$-b-vex on $\Omega \times \mathbb{R}$.

Conversely, let us take $E(g)$ is GSE $\times E^{\prime}$-b-vex on $\Omega \times \mathbb{R}$. Assume that $\mu_{1}, \mu_{2} \in \Omega, \alpha \in[0,1]$ and $\delta \in[0,1]$, then $\left(\mu_{1}, g\left(\mu_{1}\right)\right) \in E(g)$ and $\left(\mu_{2}, g\left(\mu_{2}\right)\right) \in E(g)$.

In addition, $\left(\eta_{\alpha \mu_{1}+\varepsilon\left(\mu_{1}\right), \alpha \mu_{2}+\varepsilon\left(\mu_{2}\right)}(\delta b), \delta b F\left(g\left(\mu_{1}\right)\right)+(1-\delta b) F\left(g\left(\mu_{2}\right)\right)\right) \in E(g) \Longrightarrow$

$$
\begin{aligned}
g\left(\eta_{\alpha \mu_{1}+\varepsilon\left(\mu_{1}\right), \alpha \mu_{2}+\varepsilon\left(\mu_{2}\right)}(\delta b)\right) & \leq \delta b F\left(g\left(\mu_{1}\right)\right)+(1-\delta b) F\left(g\left(\mu_{2}\right)\right) \\
& =\delta b g\left(\varepsilon\left(\mu_{1}\right)\right)+(1-\delta b) g\left(\varepsilon\left(\mu_{2}\right)\right)
\end{aligned}
$$

Hence, the result.

Theorem 3.5. Let $\left\{\Omega_{j}\right\}_{j=1, \cdots, n}$ be a family of $G S E \times F$-b-vex sets. Then $\cap_{j=1, \cdots, n} \Omega_{j}$ is also GSE $\times F$-b-vex set.

Proof. Let $\left(\mu_{1}, a_{1}\right),\left(\mu_{2}, a_{2}\right) \in \cap_{j=1, \cdots, n} \Omega_{j}$, then $\left(\mu_{1}, a_{1}\right),\left(\mu_{2}, a_{2}\right) \in \Omega_{j}, \quad \forall j . \Longrightarrow$

$$
\left(\eta_{\alpha \mu_{1}+\varepsilon\left(\mu_{1}\right), \alpha \mu_{2}+\varepsilon\left(\mu_{2}\right)}(\delta b), \delta b F\left(a_{1}\right)+(1-\delta b) F\left(a_{2}\right)\right) \in \Omega_{j},
$$


$\forall \alpha \in[0,1]$ and $\delta \in[0,1]$. Hence,

$$
\left(\eta_{\alpha \mu_{1}+\varepsilon\left(\mu_{1}\right), \alpha \mu_{2}+\varepsilon\left(\mu_{2}\right)}(\delta b), \delta b F\left(a_{1}\right)+(1-\delta b) F\left(a_{2}\right)\right) \in \cap_{j=1, \cdots, n} \Omega_{j},
$$

$\forall \alpha \in[0,1]$ and $\delta \in[0,1]$. This shows that,$\cap_{j=1, \cdots, n} \Omega_{j}$ is GSE $\times F$-b-vex set.

Theorem 3.6. Suppose that $G: \mathbb{R} \longrightarrow \mathbb{R}$ such that $G(g(x)+\mu)=g(\varepsilon(x))+\mu, \forall \mu \in \mathbb{R}, \mu \geqslant 0$. Let $\left\{g_{i}\right\}_{i \in I}$ be a family of real valued functions that is defined on a GSE-b-vex set $\Omega$ and bounded from above. Then, $g(x)=\sup _{i \in I} g_{i}(x), x \in \Omega$ is GSE-b-vex on $\Omega$.

Proof. Let $g_{i}, i \in I$ be a GSE-b-vex function on $\Omega$, then

$$
E\left(g_{i}\right)=\left\{(x, \mu): x \in \Omega, \mu \in \mathbb{R}, g_{i}(x) \leq \mu\right\}
$$

are GSE $\times F$-b-vex on $\Omega \times \mathbb{R}$. Hence,

$$
\begin{aligned}
\cap_{i \in I} E\left(g_{i}\right) & =\left\{(x, \mu): x \in \Omega, \mu \in \mathbb{R}, g_{i}(x) \leq \mu, i \in I\right\} \\
& =\{(x, \mu): x \in \Omega, \mu \in \mathbb{R}, g(x) \leq \mu\}
\end{aligned}
$$

is GSE $\times F$-b-vex set. Then, by Theorem $3.4 g$ is a GSE-b-vex function.

\section{REFERENCES}

[1] I. A. Abou-Tair and W. T. Sulaiman, Inequalities via convex functions, Int. J.Math. Sci. 22(1999), 543-546.

[2] C. R. Bector and C. Singh, B-vex functions, J. Optim. Theory Appl. 71(2) (1991), 237-253.

[3] V. Boltyanski, H. Martini and P.S. Soltan, Excursions Into Combinatorial Geometry, Springer, Berlin, 1997.

[4] L. Danzer, B. Grünbaum and V. Klee, Helly's theorem and its relatives. In: V. Klee (ed.) Convexity. Proc. Sympos. Pure Math., vol.7, pp.101-180. Amer. Math. Soc., Providence, 1963.

[5] A. Iqbal, S. Ali and I. Ahmad, On geodesic E-convex sets, geodesic E-convex functions and E-epigraphs, J. Optim. Theory Appl. 55(1)(2012), 239-251.

[6] M. A. Jiménez, G. R. Garzón and A. R. Lizana, Optimality conditions in vector optimization. Bentham Science Publishers, 2010.

[7] A. Kiliçman and W. Saleh, On geodesic strongly E-convex sets and geodesic strongly E-convex functions, J. Inequal. Appl. 2015 (2015), 297.

[8] A. Kiliçman and W. Saleh, On properties of geodesic semilocal E-preinvex functions, J. Inequal. Appl. 2018 (2018), 353.

[9] H.Martini and K.J. Swanepoel, Generalized Convexity notions and Combinatorial Geometry, Gongr. Numer. 164 (2003), 65-93.

[10] H. Martini and K.J. Swanepoel, The geometry of minkowski spaces- a survey, Part II. Expo. Math. 22 (2004), 14-93.

[11] F. Mirzapour, A. Mirzapour and M. Meghdadi, Generalization of some important theorems to E-midconvex functions, Appl. Math. Lett. 24(8) (2011),1384-1388.

[12] M. A. Noor, Fuzzy preinvex functions, Fuzzy Sets Syst. 64(1994), 95-104.

[13] M. A. Noor, K. I. Noor and M. U. Awan, Generalized convexity and integral inequalities, Appl. Math. Inf. Sci. 9(1)(2015), 233-243. 
[14] T. Rapcsak, Smooth Nonlinear Optimizatio in $\mathbb{R}^{n}$, Kluwer Academic, 1997.

[15] Y. R. Syau, Lixing Jia, and E. Stanley Lee, Generalizations of E-convex and B-vex functions, Comput. Math. Appl. 58(4) (2009), 711-716.

[16] C. Udrist, Convex Funcions and Optimization Methods on Riemannian Manifolds, Kluwer Academic, 1994.

[17] E. A. Youness, On E-convex sets, E-convex functions and E-convex programming, J. Optim.Theory Appl. 102 (1999), 439-450.

[18] E. A. Youness and Tarek Emam, Strongly E-convex sets and strongly E-convex functions, J. Interdisciplinary Math. $8(1)(2005), 107-117$.

[19] G.Y. Wang, Some Properties of strongly E-B-vex functions, Sustainable Development-Special track within SCET $2012(2012), 247$. 